\title{
REVIEWERS OF THE „ENVIRONMENTAL PROTECTION AND NATURAL RESOURCES" \\ Vol. 32 No 1(87), 2(88), 3(89), 4(90), 2021
}

\author{
Drhab. Wojciech Dmuchowski, prof. SGGW \\ Drhab. Wojciech Gotkiewicz, prof. UWM \\ Ph.D. $\quad$ Fumito Koike, Japan \\ Dr Bożena Kornatowska \\ Ph.D. Afirm Berisha, Albania \\ Drhab. Grażyna Porębska, \\ Prof. drhab.inz. $\quad$ Lech Łobocki \\ Dr Anna Dabrowska \\ Drhab. Beata Kuziemska, prof. UTH w Siedlcach \\ Drhab.inż. Z Zbigniew Karaczun, prof. SGGW \\ Drhab. Grażyna Wojtkowska-Łodej, prof SGH \\ Drhab. Magdalena Florek-Łuszczki, prof. IMW \\ Assistant Professor Engida Esayas Dube, Ethiopia \\ Ph.D. Asnake Yimam, Ethiopia \\ Drhab. Piotr Jeżowski, prof. SGH \\ Dr Andrzej Tarłowski \\ Drinż. Ewelina Siwiec \\ Drhab.inż. Grażyna Cacak-Pietrzak \\ Drinż. Krystian Szczepański \\ Dr Elżbieta Goryńska-Goldmann \\ Drhab.inż. Anna Sylwia Tarczyńska, prof. UWM \\ Prof.drhab. Anna Lewicka-Strzałecka \\ Drhab.inż. Beata Bilska \\ Drhab.inż. Mariola Kwasek, prof. IERiĠ̇-PIB \\ Drinż. Sylwia Łaba \\ Prof.drhab. Barbara Gworek \\ Drhab. Alicja Walosik, prof. UP w Krakowie \\ Dr Jan Pietroń \\ Ph.D. Muhammad Tayyab, China \\ Prof.drhab. Axel Schwerk
}

The editors would like to publicly acknowledge the people who served as reviewers on the journal during 2021. Without their efforts, the quality of the journal could not be sustained 